\title{
A Context-Aware Edge-Based VANET Communication Scheme for ITS ${ }^{\dagger}$
}

\author{
Chang An ${ }^{1, *}$, Celimuge $\mathrm{Wu}^{2, *}$ (1) , Tsutomu Yoshinaga ${ }^{2}$, Xianfu Chen ${ }^{3}$ and Yusheng $\mathrm{Ji}^{4}$ \\ 1 Inner Mongolia Normal University, Hohhot 010010, China \\ 2 Department of Computer and Network Engineering, The University of Electro-Communications, \\ Tokyo 182-8585, Japan; yoshinaga@uec.ac.jp \\ 3 VTT Technical Research Centre of Finland, FI-90571 Oulu, Finland; Xianfu.Chen@vtt.fi \\ 4 Information Systems Architecture Research Division, National Institute of Informatics, \\ Tokyo 101-8430, Japan; kei@nii.ac.jp \\ * Correspondence: 20060623@imnu.edu.cn (C.A.); celimuge@uec.ac.jp (C.W.); Tel.: +81-42-443-5635 (C.W.) \\ $+\quad$ This paper is an extended version of our paper published in $\mathrm{Wu}, \mathrm{C}$.; Yoshinaga, T.; Ji, Y. Context-aware \\ Unified Routing for VANETs Based on Virtual Clustering. In Proceedings of the 2016 IEEE 27th Annual \\ International Symposium on Personal, Indoor, and Mobile Radio Communications (PIMRC), Valencia, Spain, \\ 4-8 September 2016.
}

Received: 22 May 2018; Accepted: 21 June 2018; Published: 24 June 2018

check for updates

\begin{abstract}
We propose a context-aware edge-based packet forwarding scheme for vehicular networks. The proposed scheme employs a fuzzy logic-based edge node selection protocol to find the best edge nodes in a decentralized manner, which can achieve an efficient use of wireless resources by conducting packet forwarding through edges. A reinforcement learning algorithm is used to optimize the last two-hop communications in order to improve the adaptiveness of the communication routes. The proposed scheme selects different edge nodes for different types of communications with different context information such as connection-dependency (connection-dependent or connection-independent), communication type (unicast or broadcast), and packet payload size. We launch extensive simulations to evaluate the proposed scheme by comparing with existing broadcast protocols and unicast protocols for various network conditions and traffic patterns.
\end{abstract}

Keywords: vehicular networks; VANET; edge computing; unicast communications; broadcast communications; context-aware communications; intelligent transportation systems

\section{Introduction}

Due to the rapid increase of the number of cars in China, people are facing increasing traffic congestion, car accidents, and $\mathrm{CO}_{2}$ pollution, as well as longer commuting time and increasing difficulty in finding parking. The intelligent transportation systems (ITS) become more and more important for supporting day-to-day commuter mobility management, tourism industry as well as large city event (e.g., events such as football matches, music events, or even bigger events like Olympic games) or even at unexpected events (e.g. flood, earthquake, etc.). All these applications require an efficient networking architecture to collect/disseminate information from/to vehicles, and other sensors/actuators. Vehicular ad hoc networks (VANETs) are capable of utilizing decentralized communication technologies to support efficient collection and dissemination of traffic related information. However, due to the limited wireless resources, high mobility of vehicles, and possible large node density, it is difficult to provide a satisfactory throughput with the conventional networking technologies.

Recently, mobile edge computing is introduced to conduct computation, caching task at the edge nodes that are closer than the cloud in order to reduce the response delay and improve the throughput. 
Some studies have proposed the use of vehicles as edge nodes [1-4]. However, the existing works do not sufficiently discuss the selection of edge nodes in a decentralized network environment. The joint operation of route selection and edge selection is not discussed as well.

There have been many studies discussing the routing problem in VANETs [5-20]. However, the unicast routing problem and broadcast problem have been discussed separately. Different applications could possibly require different levels of quality of services (QoS). Therefore, it is particularly important to consider context information, such as communication type (unicast or broadcast), end-to-end connection-dependency, and packet payload size, in the route selection. In the unicast communications, frame retransmissions can be conducted at the MAC layer while this is impossible for broadcast communications. As a result, the edge node selection criteria for unicast and broadcast communications are totally different. If an application only cares about the throughput, a delay tolerant approach could be a solution [21]. The packet payload size is another important context information that should be considered for the route selection. A larger packet will face a higher packet loss probability. The performance of a communication system can be improved by taking into account the context information for the routing decision. However, the design of an edge-based approach that is capable of handling different types of applications with different context information is still an under-explored research issue.

In this paper, we propose a context-aware edge-based general communication scheme for data exchange in vehicular networks. The proposed scheme uses a distributed approach to select edge nodes which are responsible to conduct packet forwarding. As compared with the conventional approach, the edge-based approach could efficiently reduce the number of concurrent sender nodes by aggregating user traffics at the edge nodes (Here the number of concurrent sender nodes denotes the number of nodes that content for the same wireless resources). When different devices acquire the same content, content cashing can be easily achieved at edge nodes. Since edge nodes are selected by considering the context information of the communications, the proposed scheme is able to handle various traffic patterns including unicast communications and broadcast communications. This paper is an extension of our previous conference paper [22]. This paper improves [22] by introducing a multi-hop data virtualization approach for connection-independent communications, and also presents new simulation results to show the performance advantage of the proposed approach over existing studies in a more comprehensive manner. The main contributions of this paper are as follows.

- We propose a context-aware general edge-based scheme that is possible to handle different types of communications with different requirements.

- The proposed scheme employs a fuzzy logic-based edge node selection protocol to find the best edge nodes in a decentralized network, which can achieve an efficient use of wireless resources by conducting packet forwarding through edges.

- The proposed scheme also uses a reinforcement learning algorithm to learn the best route for the last two-hop communications.

- We launch extensive simulations to evaluate the proposed scheme by comparing with other baselines.

\section{Related Work}

The existing studies cover broadcast protocols, unicast routing protocols, and vehicular edge computing approaches. Different types of communications with different context information possibly require different edges. An unified solution is required to handle different types of communications efficiently. However, there is no research addressing the design of context-aware route selection with edge assistance. 


\subsection{Vehicular Edge Computing}

He et al. [1] have formulated the resource allocation strategy in vehicular networks as a joint optimization problem where the networking, caching and computing are taken into consideration. However, how to achieve the optimization in a decentralized network is not seriously addressed. Yuan et al. [2] have demonstrated that content caching at base stations and autonomous vehicles could improve the content delivery performance. Jutila [3] has proposed an admission control approach and a flow scheduling mechanism that work on network edges to optimize and control traffic flows and network resources. Wang et al. [4] have proposed a collaborative vehicular edge computing framework where a software defined network-based approach is employed to program, manipulate and configure network in a logically centralized way. While existing studies mainly focus on the use of caching and computation technologies to improve the networking performance, the joint route selection and edge selection problem in a decentralized network is not discussed adequately.

\subsection{VANET Multi-Hop Broadcast Communications}

A pioneer work on multi-hop broadcast protocols can be found in [5] where three different receiver-oriented approaches are proposed. Lai et al. [6] have proposed an average packet loss rate analysis model for vehicle-to-vehicle multi-hop broadcasting taking into account the mobility of vehicles, wireless channel conditions, and media access control (MAC). Rodriguez et al. [7] have proposed a streamwise queuing system on the receiver side to filter out the unimportant messages in a congestion situation. Alotaibi et al. [8] have proposed area-based dissemination protocols in vehicular networks with heterogeneous transmission powers. In the area-based dissemination protocols, a node that has higher potential new coverage area transmits first. Bi et al. [8] have proposed a protocol that takes into account the road layout in the forwarding decision, where three different types of broadcast decisions, namely the directional broadcast, bi-directional broadcast and multi-directional broadcast, are defined. A network coding-based approach to reduce the broadcast overhead has been proposed in [10]. Nguyen et al. [11] have proposed a hybrid TDMA/CSMA protocol that adjusts the length of broadcast frames based on three-hop neighbor information.

\subsection{VANET Multi-Hop Unicast Communications}

Many protocols have been proposed to take into account the vehicle mobility, route length, and inter-vehicle wireless link quality for the route selection since the performance of a communication route is affected by these multiple factors. Goudarzi et al. [14] have proposed a traffic-aware position-based routing protocol for city environments. The protocol uses small control packets to sample traffic conditions and makes routing decisions based on congestion conditions. In [15], the regularity of vehicle moving behaviors has been used to improve the routing performance by predicting a vehicle's future locations based on the past traces and a hidden Markov model. Darwish et al. [16] have discussed the routing decision at the intersection, and proposed a protocol that makes routing decision based on road structure, vehicle position and received signal strength. There have been several studies considering some special types of vehicles to improve the routing. Sun et al. [18] have proposed a bus-based street-centric routing approach that uses buses as the main relay nodes for the packet forwarding. Similarly, Zhang et al. [19] uses the bus systems as routing backbones of VANETs. In [20], drones are used for boosting VANET communications.

However, these existing approaches do not adequately address the MAC layer contention issue in a high-density network. Since vehicles could be deployed in a high-density manner for some hours or some road segments, the number of concurrent sender nodes is expected to be large. IEEE 802.11p, the standard for wireless access in vehicular environments, has the performance degradation problem when the number of sender nodes increases due to the MAC layer contention scheme based on the exponential backoff $[12,13]$. However, this problem is not sufficiently considered in the design of routing protocols. 


\section{Propose Scheme}

\subsection{Assumptions and Design Principles}

Each vehicle (node) is able to get its own position information and road map through positioning services. Hello messages are exchanged periodically ( $1 \mathrm{~Hz}$ by default) to share location information and velocity information between neighbors.

The proposed scheme uses edge nodes to forward data packets. To efficiently utilize wireless resources, the scheme uses the same edge nodes for different traffic flows as far as possible. However, as different applications could expect different levels of QoS, the scheme also employs a context-aware approach to differentiate the traffic flows with different requirements. The proposed scheme generates different virtual edge nodes for different traffic flows with different context information. While the conventional routing protocols use the same route for the traffic flows with the same source-destination pairs, the proposed scheme could possibly use different routes as the edge nodes depending on the context information. We explain the proposed scheme by using three different types of communication patterns, namely end-to-end connection-dependent unicast, end-to-end connection-independent broadcast, and end-to-end connection-independent unicast communications.

\subsection{Edge Node Selection and Packet Forwarding with Edge Nodes}

Here, vehicles are used as edge nodes which conduct packet forwarding, computing, and content caching. We propose a concept that uses the same forwarder nodes for different traffic flows. As shown in Figure 1, three different traffic flows, specifically "P1 $\rightarrow$ R1", "P2 $\rightarrow$ R2", and "P2 $\rightarrow$ R2-2", use the same edges nodes for packet forwarding. The use of the same forwarder nodes results in two main benefits. First, the efficiency of wireless resource utilization is improved because the number of sender nodes can be reduced. Second, content caching at edge nodes becomes easier, resulting in a higher performance.

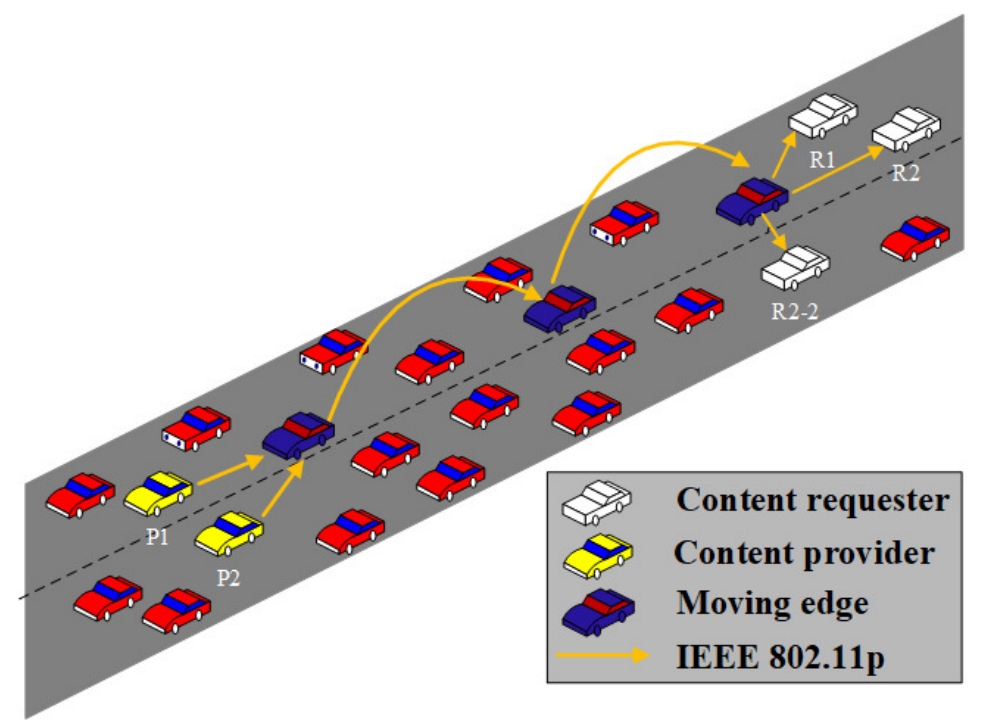

Figure 1. Edge-based forwarding.

\subsection{Criteria for Edge Node Selection}

The proposed scheme selects the edge nodes using a distributed approach. The vehicle velocity, the vehicle distribution, and the channel conditions (link qualities) between the edge nodes and ordinary nodes are taken into account for the edge node selection. The consideration of vehicle velocity is to select slower vehicles as possible in order to avoid the overhead that could possibly occur due to the frequent change of edge nodes. The vehicle distribution is considered using the number of 
neighbors moving to the same direction which can reflect the long-term vehicle movement (in a two-way road, the edge nodes should be selected from the vehicles that have more vehicles moving toward the same direction). The link quality is also an important metric because a node that has better connections (for example, higher antenna height) with other non-edge nodes is preferred. A fuzzy logic-based approach is used to integrate these three metrics in the evaluation of the fitness for being an edge node.

In the proposed scheme, the vehicle velocity and the number of neighbor vehicles driving to the same direction are exchanged by hello messages. Each node calculates a competency value (as being an edge) for itself and each neighbor vehicle. If the node has the largest competency value in its vicinity $\left(\frac{R}{2}\right.$ where $R$ is the average transmission range), the node claims itself as an edge node in the next hello message. Edge node selection is conducted on a road segment basis which is defined in [23]. This ensures the connectivity between the selected edge nodes.

\subsection{Calculation of Competency Value Based on Fuzzy Logic}

A node becomes an edge node when it has the largest competency value in the neighborhood. A fuzzy logic-based algorithm is used to evaluate the competency value.

\subsubsection{Procedure}

For each one-hop neighbor node, a node calculates a competency value as follows.

- Calculation of the three factors: Calculate the vehicle velocity, the number of vehicles moving to the same direction, and the channel condition information mentioned before.

- Fuzzification: Use predefined linguistic variables and membership functions to convert the factors to the corresponding fuzzy values.

- Mapping and IF/THEN rule-based evaluation: Map the fuzzy values to the predefined IF/THEN rules and combine the rules to get the rank of the neighbor as a fuzzy value.

- Defuzzification: Use a predefined output membership function and defuzzification method to convert the fuzzy value to a numerical value.

\subsubsection{Calculation of Multiple Factors}

Upon reception of a hello message from a neighbor $x$, node $s$ calculates the following factors.

Velocity Factor $(V F)$ : Node $s$ extracts the velocity of node $x, v(x)$, and calculates $\operatorname{VF}(s, x)$ (the velocity factor for node $x$ calculated at node $s$ ) as

$$
V F(s, x)=\frac{|v(x)|-\min _{y \in N_{s}}|v(y)|}{\max _{y \in N_{s}}|v(y)|}
$$

where $N_{s}$ is the set of neighbors for node $s$. A lower $V F$ indicates a lower velocity. $V F$ is updated for every hello interval using a weighted exponential moving average as

$$
V F_{i}(s, x) \leftarrow(1-\alpha) \times V F_{i-1}(s, x)+\alpha \times V F_{i}(s, x),
$$

where $V F_{i-1}(s, x)$ and $V F_{i}(s, x)$ denote the previous $V F$ value and current $V F$ value respectively. $V F$ is initialized to 1 . The coefficient $\alpha$ is set to 0.7 which is the best value for most situations according to our simulation results [the same value is used in Equations (4) and (5)].

Follower Density Factor (FDF): Node $x$ announces the number of neighbor vehicles $(c(x))$ driving to the same direction by using hello messages. FDF of node $x$ is calculated as

$$
F D F(s, x)=\frac{c(x)}{\max _{y \in N_{s}} c(y)}
$$


FDF indicates the vehicle density moving towards the same direction. A higher FDF is preferable as it means that the node is more suitable for being an edge node. FDF is initialized to 0 , and updated for each hello interval using a weighted exponential moving average as

$$
F D F_{i}(s, x) \leftarrow(1-\alpha) \times F D F_{i-1}(s, x)+\alpha \times F D F_{i}(s, x)
$$

Channel Condition Factor (CCF): For simplicity, the hello packet reception ratio is used as the channel Condition Factor (CCF). We only calculate the message reception ratio of the hello messages sent by the nodes located in $R$ where $R$ is the average transmission range. The hello messages are sent periodically with a predefined interval ( $1 \mathrm{~s}$ by default). If a vehicle has a better channel condition than other vehicles (for example, a truck with higher antenna), the CCF is larger. The CCF is initialized to 0 , and updated as

$$
C C F_{i}(s) \leftarrow(1-\alpha) C C F_{i-1}(s)+\alpha \times C C F_{i}(s)
$$

\subsubsection{Fuzzification}

Figure 2 shows the fuzzy membership functions for the velocity factor, follower density factor and channel condition factor. The velocity membership function defines what degree the velocity factor belongs to \{Slow, Medium, Fast\}. Similarly, the follower density membership function defines what degree belongs to \{Heavy, Medium, Light\} and what degree the channel condition factor belongs to $\{$ Good, Medium, Bad\}.
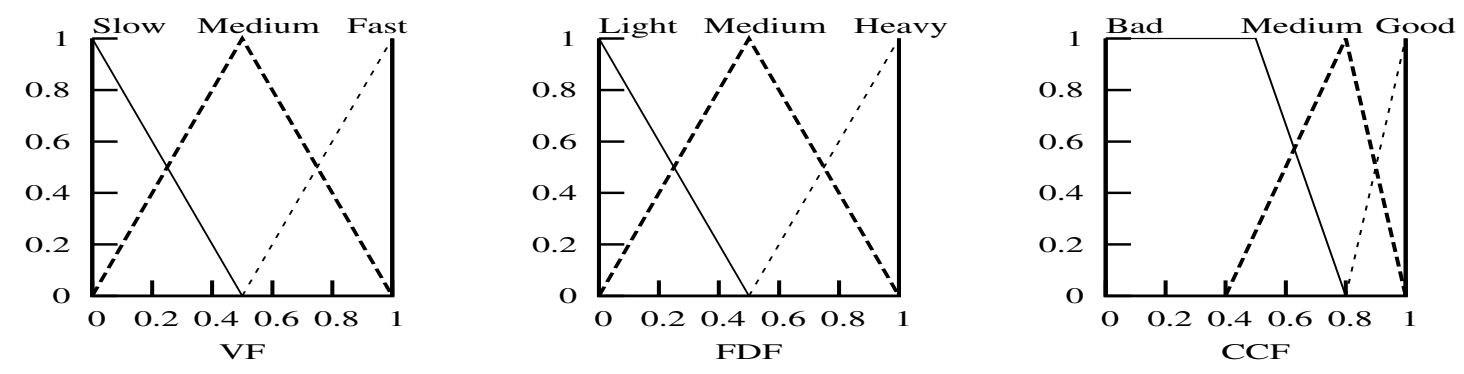

Figure 2. Fuzzy membership functions ((left): $V F$, (middle): $F D F$, (right): $C C F)$.

\subsubsection{Mapping and Combination of IF/THEN Rules}

Each node calculates the rank of the vehicle based on the IF/THEN rules (see Table 1). Since multiple rules could apply at the same time, the Min-Max method is used to combine their evaluation results (the same as the method used in [23]).

\subsubsection{Defuzzification}

The output membership function used is as shown in Figure 3. The Center of Gravity (COG) method is used in the defuzzification.

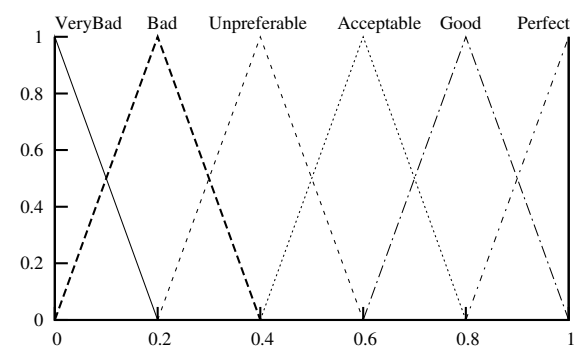

Figure 3. Output membership function. 
Table 1. Rule Base.

\begin{tabular}{ccccc}
\hline & Velocity & Follower Density & Channel Condition & Rank \\
\hline Rule1 & Slow & Heavy & Good & Perfect \\
Rule2 & Slow & Heavy & Medium & Good \\
Rule3 & Slow & Heavy & Bad & Unpreferable \\
Rule4 & Slow & Medium & Good & Good \\
Rule5 & Slow & Medium & Medium & Acceptable \\
Rule6 & Slow & Medium & Bad & Bad \\
Rule7 & Slow & Light & Good & Unpreferable \\
Rule8 & Slow & Light & Medium & Bad \\
Rule9 & Slow & Light & Bad & VeryBad \\
Rule10 & Medium & Heavy & Good & Good \\
Rule11 & Medium & Heavy & Medium & Acceptable \\
Rule12 & Medium & Heavy & Bad & Bad \\
Rule13 & Medium & Medium & Good & Acceptable \\
Rule14 & Medium & Medium & Medium & Unpreferable \\
Rule15 & Medium & Medium & Bad & Bad \\
Rule16 & Medium & Light & Good & Bad \\
Rule17 & Medium & Light & Medium & Bad \\
Rule18 & Medium & Light & Bad & VeryBad \\
Rule19 & Fast & Heavy & Good & Unpreferable \\
Rule20 & Fast & Heavy & Medium & Bad \\
Rule21 & Fast & Heavy & Bad & VeryBad \\
Rule22 & Fast & Medium & Good & Bad \\
Rule23 & Fast & Medium & Medium & Bad \\
Rule24 & Fast & Medium & Bad & VeryBad \\
Rule25 & Fast & Light & Good & Bad \\
Rule26 & Fast & Light & Medium & VeryBad \\
Rule27 & Fast & Light & Bad & VeryBad \\
\hline & & & & \\
\hline
\end{tabular}

\subsection{Last Two-Hop Route Optimization with Reinforcement Learning}

The proposed scheme uses edge nodes to forward packets. As a result, different traffic flows could use the same edge nodes to forward packets assuming that the context information is the same (packet size and transmission type). This is efficient for utilizing wireless resources by reducing the number of sender nodes. However, this could increase the number of hops if the source (destination) node is very close from the next/previous edge node. To make the routing more efficient, we utilize a reinforcement learning-based algorithm to optimize the last two-hop route from/to the source/destination node. As shown in Figure 4, since E1, E2, E3, and E4 are the edge nodes, the default route from the source node (S) to the destination node (D) is " $\mathrm{S} \rightarrow \mathrm{E} 1 \rightarrow \mathrm{E} 2 \rightarrow \mathrm{E} 3 \rightarrow \mathrm{E} 4 \rightarrow \mathrm{D}$ ". By using the last two-hop optimization, the route can be optimized to " $\mathrm{S} \rightarrow \mathrm{F} 1 \rightarrow \mathrm{E} 2 \rightarrow \mathrm{E} 3 \rightarrow \mathrm{F} 2 \rightarrow \mathrm{D}$ " which is more efficient than the default route.

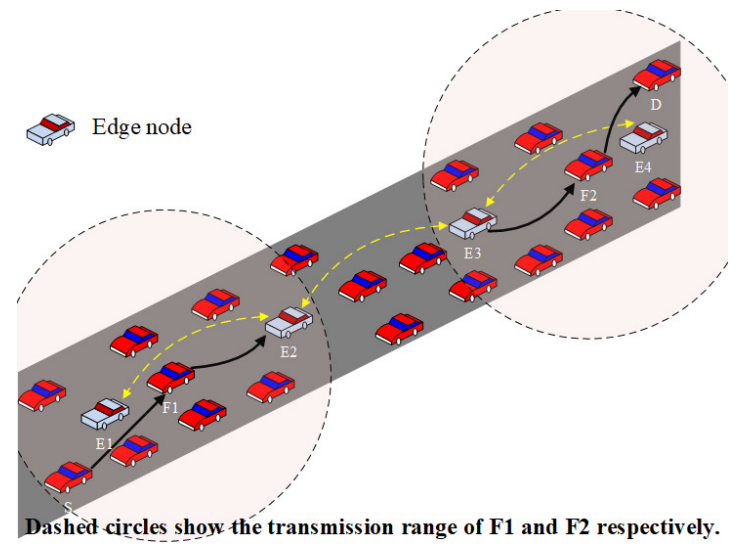

Figure 4. Last two-hop optimization. 


\subsubsection{Q-Learning Model}

We define the $Q$-Learning algorithm as follows (Table 2). The entire network is the environment. Each packet $P(o, r)$, indexed by its originator node $o$ and the reference node $r$ (the destination node or an edge node) is an agent. A node selects the next hop that it should forward a packet to. Hence the possible set of actions allowed at the node is the set of one-hop neighbors. Every node maintains a $Q$-Table which consists of $Q$-value $[Q(r, x)]$ whose value ranges from 0 to 1 , where $x$ is the next hop to the reference node.

Table 2. Q-learning model.

\begin{tabular}{ll}
\hline Environment & The Entire Vehicular Ad Hoc Network \\
\hline Agent & Each packet $P(o, r)$ \\
State of the agent & Each node in the network \\
State space & The set of all nodes in the network \\
Actions & Selecting an one-hop neighbor as the next hop \\
\hline
\end{tabular}

\subsubsection{Update of $Q$-Values}

For each one-hop neighbor, a node maintains a value, $l s(c, x)$, which shows the link status between node $c$ and $x$. For simplicity, we use hello reception ratio to estimate the link status. However, the estimation can be improved by taking into account the vehicle velocity in the link status evaluation. The $Q$-Table is updated upon the reception of hello messages. Each node needs to maintain a $Q$-value for each one-hop neighbor, the destination (the traffic source node for the TCP ACK messages) node, and the edge nodes located in two-hop distance. $Q$-values are broadcasted by each node using hello messages. Each $Q$-value is initialized to 0 . Upon reception of a hello message from node $x$, node $c$ updates the corresponding $Q$-value to the node $r$ as

$$
\begin{aligned}
Q_{c}(r, x) \leftarrow \quad & \hat{\alpha} \times l s(c, x) \times\left\{\hat{R}+\gamma \times \max _{y \in N_{x}} Q_{x}(r, y)\right\} \\
& +(1-\hat{\alpha}) \times Q_{c}(r, x) .
\end{aligned}
$$

The learning rate $(\hat{\alpha})$ is 0.7 , and the discount factor $(\gamma)$ is 0.9 . $\max _{y \in N_{x}} Q_{x}(r, y)$ is the maximal $Q$-value of $x$ to node $r$. The reward $\hat{R}$ is calculated as

$$
\hat{R}= \begin{cases}1, & \text { if } c \in N_{r} \\ 0, & \text { otherwise }\end{cases}
$$

where $N_{r}$ denotes the one-hop neighbor set of node $r$. When node $c$ is a neighbor of node $r$, the reward is 1 and otherwise 0 . Please note that only one $Q$-value is maintained for each pair of state and action. Upon reception of a hello message, the corresponding $Q$-value is updated according to Equation (6) which discounts the reward with the increase of number of hops. This ensure that the hop count is considered in the route selection. The quality of each link is also taken into account by discounting the reward with the link status value $[l s(c, x)]$. As a result, a $Q$-value represents the evaluation of a next packet forwarder candidate with the consideration of multi-hop performance. Therefore, the proposed scheme is able to optimize the route to the reference node. Figure 5 shows an example for $Q$-value update where $l s(\cdot, \cdot)$ is 1. 


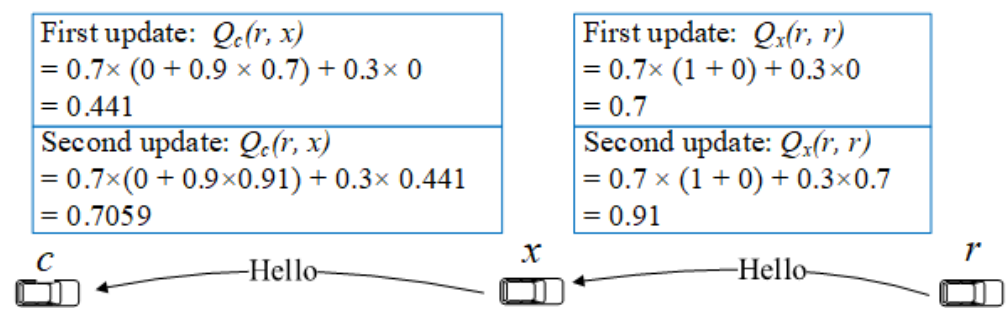

Figure 5. An example for $Q$-value update.

\subsection{Context Acquisition and Utilization}

Three different types of context information, namely connection-dependency (connection-dependent or connection-independent), communication type (unicast or broadcast), and packet payload size, are considered in the proposed scheme. More context information can be included with a simple extension. Figure 6 shows the MAC broadcast frame reception ratio for various packet payload sizes, where the data are achieved by conducting a real-world VANET experiment with IEEE $802.11 \mathrm{~b} / \mathrm{g} / \mathrm{n}$ devices. It is easy to observe the significant effect of packet size on the packet reception probability.

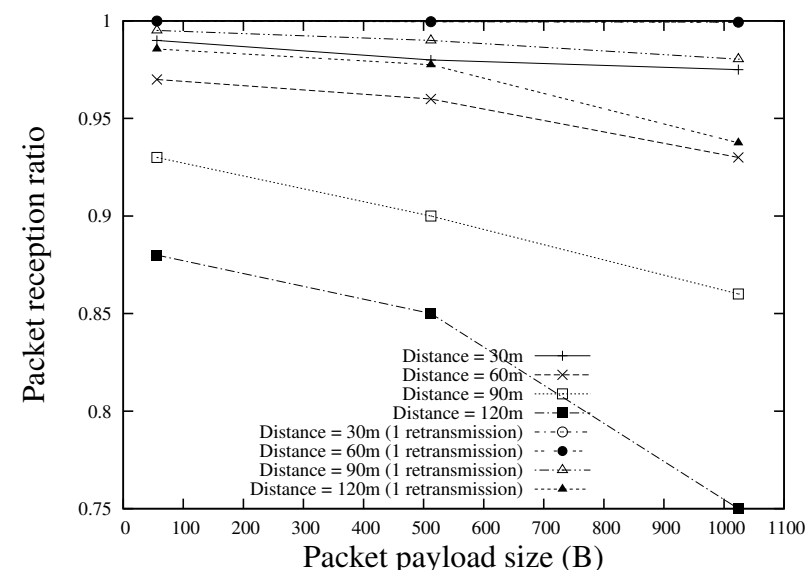

Figure 6. MAC broadcast frame reception ratio for various packet payload sizes.

VANET applications can be classified into three different categories, specifically end-to-end connection-dependent unicast, end-to-end connection-independent broadcast, and end-to-end connection-independent unicast applications. Different applications have different requirements. Specifically, for broadcast applications, since there is no retransmissions at the MAC layer, a more reliable link between two communication nodes is required. For unicast applications, the requirement on the link quality could be looser than broadcast as frame retransmissions are possible to improve the reliability.

In the proposed scheme, three different sizes of hello (probe) packets are used to estimate the frame reception probability for each size. The hello message size is randomly selected from $\{56,512,1024\}$ (bytes) (this can be easily extended to support various payload sizes). This selection lasts for $100 \mathrm{~s}$ (This is a system parameter which could be tuned according to the network environment. If the node density is not sufficiently high, this value should be set to a smaller one, which is better to calculate the reception ratios for different sizes of hello packets in a short period. Please note that the value we use here (100 s) is acceptable for most scenarios according to our simulations, and the dynamic tuning of this parameter is beyond the scope of this paper). If the selected payload size is not sufficient to transmit all the data required, the node uses multiple packets to transmit. If the selected size is larger than the size required, the node uses zero padding. Link quality information is maintained for 
each possible hello payload size $(\{56,512,1024\})$ (bytes). Upon reception of a hello message, the link quality information is updated for the corresponding payload size. For unicast applications, the hello reception ratio (CCF in Equation (5)) is calculated by considering three retransmissions at the MAC layer. By using different sizes of hello packets, we can utilize the context information to improve the efficiency of packet forwarding process (see Figure 7).

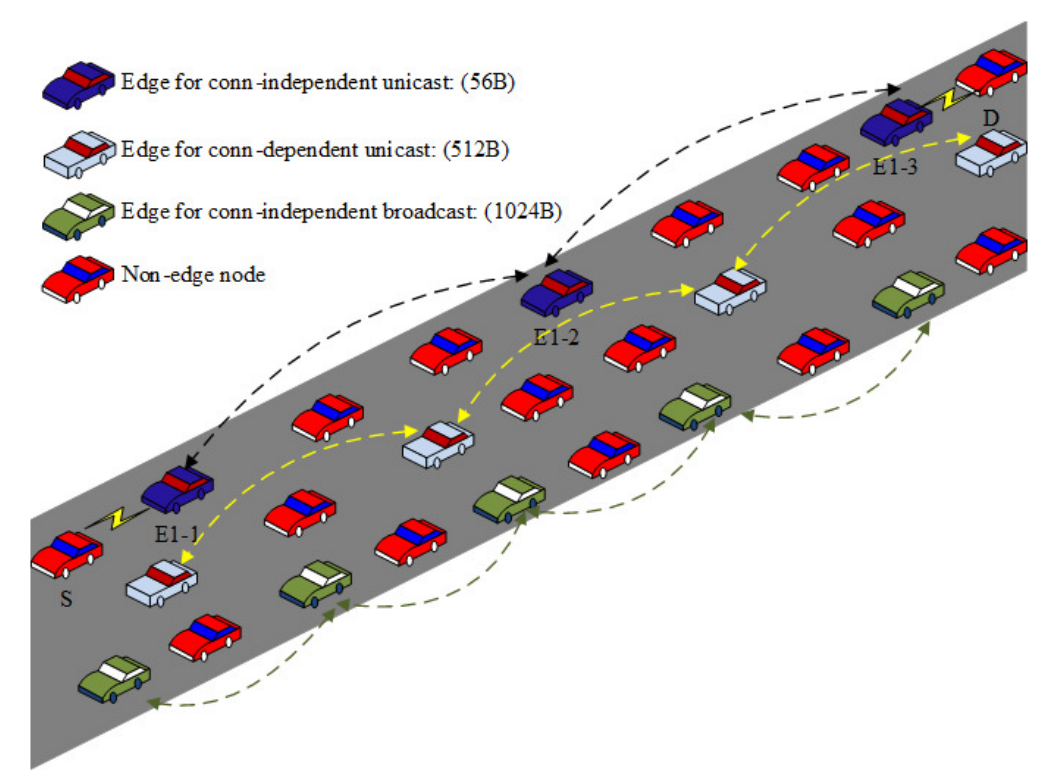

Figure 7. Context-aware edge selection.

\subsection{Virtual Multi-Hop Data Delivery for Connection-Independent Unicast Applications}

Most unicast applications in vehicular networks, such as software upgrading, car sensor data collection and so on, require a reliable end-to-end data delivery. TCP is a widely used end-to-end acknowledgment-based data delivery approach. In TCP, the sender node adapts the sending rate, namely congestion window size, by using a congestion avoidance algorithm according to the acknowledgment packets successfully received. The throughput of a TCP connection is affected significantly by the end-to-end packet loss probability. As a result, TCP has a performance degradation problem in a lossy multi-hop networks where a high end-to-end packet loss ratio restricts the increase of the congestion window size at the sender node.

The main purpose of sensor data collection applications is to send the data to the cloud. Therefore, in most cases, a real-time end-to-end feedback is not required. To solve the performance degradation problem of TCP in multi-hop networks, we use a multi-hop data delivery virtualization approach [24] to conduct multi-hop data transmissions through multiple one-hop TCP sessions. The virtualization approach works on the top of transport layer. As shown in Figure 8, a multi-hop data transmission from vehicle 1 to vehicle $\mathrm{N}$ is conducted by multiple one-hop data transmissions where each hop is managed by a TCP session, which ensures the reliability and fairness while facilitating an efficient use of wireless resources. Each forwarder node is responsible for transmitting data segments to the next hop which is closer to the destination. Since each one-hop communication is conducted based on TCP, the congestion window size at each TCP sender node is not affected by the number of hops between the source node and the destination node. 


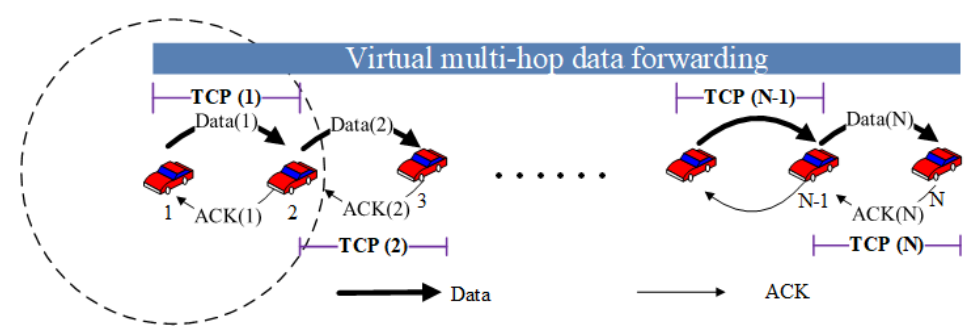

Figure 8. Virtual multi-hop data forwarding (dashed circle shows the transmission range).

\section{Simulation Results}

Network simulator ns-2.34 [25] was used to conduct simulations in street scenarios (see Table 3). The channel fading was addressed by using Nakagami propagation model (see Table 4) [26]. The average transmission distance was approximately $250 \mathrm{~m}$. The proposed scheme was compared with "Non-edge" (the conventional approach without edge computing), "Edge (BeaconSz = 56 B)" (Edge-based approach with beacon size of 56 bytes), "Edge $($ BeaconSz $=512 \mathrm{~B})$ ", and "Edge $($ BeaconSz $=1024 \mathrm{~B})$ ". The protocols were evaluated under broadcast traffic flows, connection-dependent unicast traffic flows, and connection-independent traffic flows. The error bars in the following figures indicate the $95 \%$ confidence intervals.

Table 3. Simulation Environment.

\begin{tabular}{ll}
\hline & Street Scenario \\
\hline Topology & $1700 \mathrm{~m} \times 1700 \mathrm{~m}$ \\
Number of vehicles & 619 \\
Mobility generator & SUMO [27] + TraNS [28] \\
Packet size & randomly selected from $\{56 \mathrm{~B}, 512 \mathrm{~B}, 1024 \mathrm{~B}\}$ \\
Broadcast data rate & 15 packets per second \\
MAC & IEEE 802.11p (broadcast: 3 Mbps, unicast: 27 Mbps) \\
Propagation model & Nakagami Model \\
Simulation time & $500 \mathrm{~s}$ \\
\hline
\end{tabular}

Table 4. Parameters of Nakagami Model.

\begin{tabular}{ccccc}
\hline gamma & gamma1 & gamma2 & d0_gamma_ & d1_gamma_ \\
\hline 2.0 & 2.0 & 2.0 & 200 & 500 \\
$\mathrm{~m} 0_{-}$ & $\mathrm{m} 1_{-}$ & $\mathrm{m} 2_{-}$ & $\mathrm{d} 0_{-} \mathrm{m}_{-}$ & $\mathrm{d} 1_{-} \mathrm{m}_{-}$ \\
1 & 1 & 1 & 80 & 200 \\
\hline
\end{tabular}

\subsection{Connection-Dependent Broadcast Flows}

Figure 9 shows the end-to-end packet delivery ratio for various source-destination distances. All the edge-based approaches achieve significantly better performance than the conventional approach without edge computing. This is because the packet forwarding through the edge node could efficiently reduce the MAC layer contention by using smaller numbers of sender nodes, resulting in a better wireless resource utilization. When the beacon size is small ("Edge (BeaconSz $=56 \mathrm{~B}$ )"), it is difficult to achieve a high packet delivery ratio as the small size beacon overestimates the link quality. Since the proposed scheme uses different sizes of beacons for different requirements, the link quality is evaluated more accurately, and this contributes to a significant improvement of packet delivery ratio as compared with other approaches that use constant packet size for beacons. 


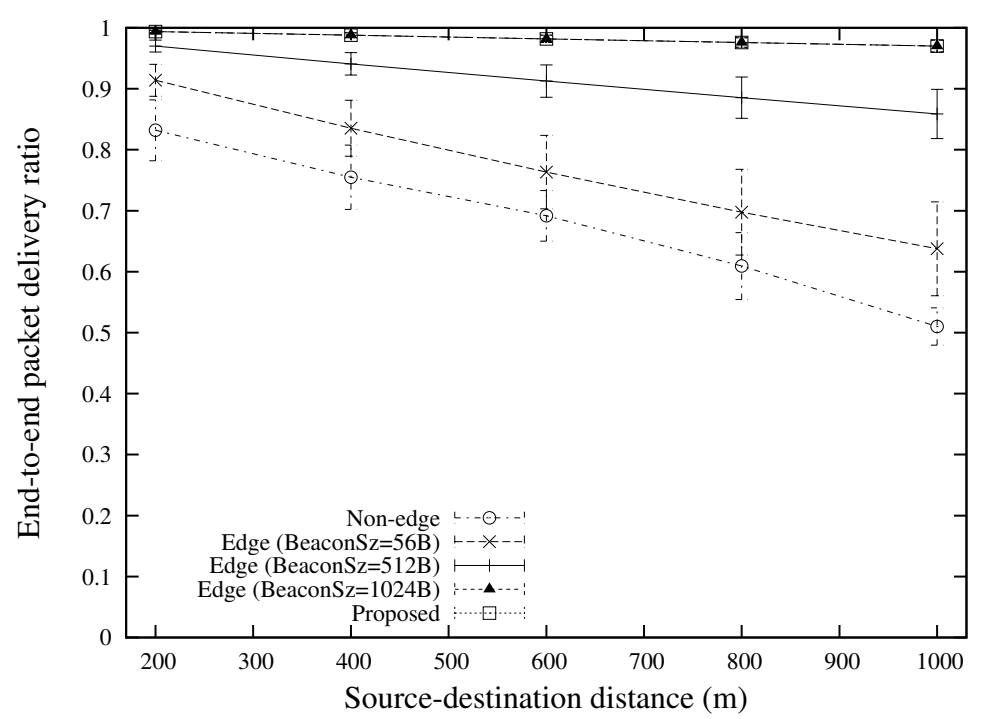

Figure 9. End-to-end packet delivery ratio for various source-destination distances (the case of 2 broadcast flows).

The end-to-end packet delivery ratio for different numbers of concurrent unicast traffic flows is shown in Figure 10. Although "Edge (BeaconSz $=1024 \mathrm{~B})$ " is able to provide a high packet delivery ratio, the performance drops drastically with the number of traffic flows as a large size beacon underestimates the link quality, resulting in a large number of hops than the optimal one. The proposed scheme is able to attain a better performance in various number of traffic flows by selecting routes with consideration of packet payload sizes. Another contributor for the high packet delivery ratio is the edge-based packet forwarding approach which could reduce the number of forwarder nodes as compared with the conventional non-edge approaches.

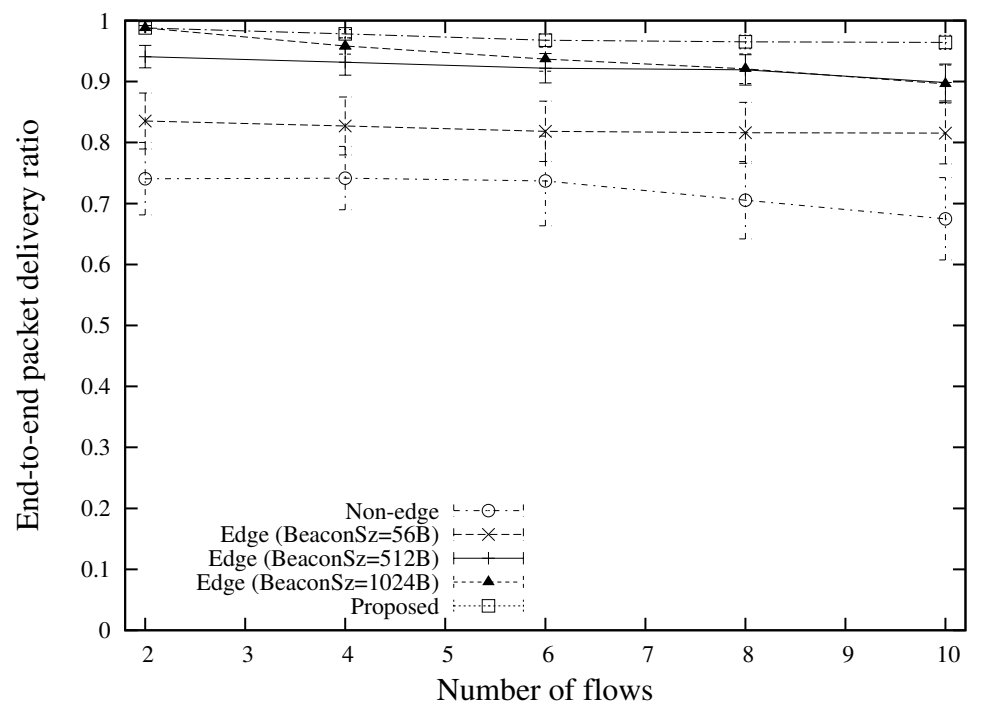

Figure 10. End-to-end packet delivery ratio for various numbers of flows (the case of $400 \mathrm{~m}$ source-destination distance).

\subsection{Connection-Dependent Unicast Flows}

The TCP throughput under various source-destination distances and various numbers of flows are shown in Figures 11 and 12, respectively. The best performance is attained by the proposed 
scheme. This is because by using different types of beacon messages, the proposed scheme can reduce the number of hops as compared with "Edge (BeaconSz $=1024 \mathrm{~B})$ " and select better forwarder nodes that have better qualities as compared with "Edge (BeaconSz $=56 \mathrm{~B})$ ". For an end-to-end TCP flow, the proposed scheme is able to select different routes for data packets and ACK packets, which can significantly shorten the round trip time as compared with the conventional approaches. The advantage over other approaches for a large number of traffic flows comes from the edge-based forwarding scheme which reduces the number of contender nodes for the same wireless resources.

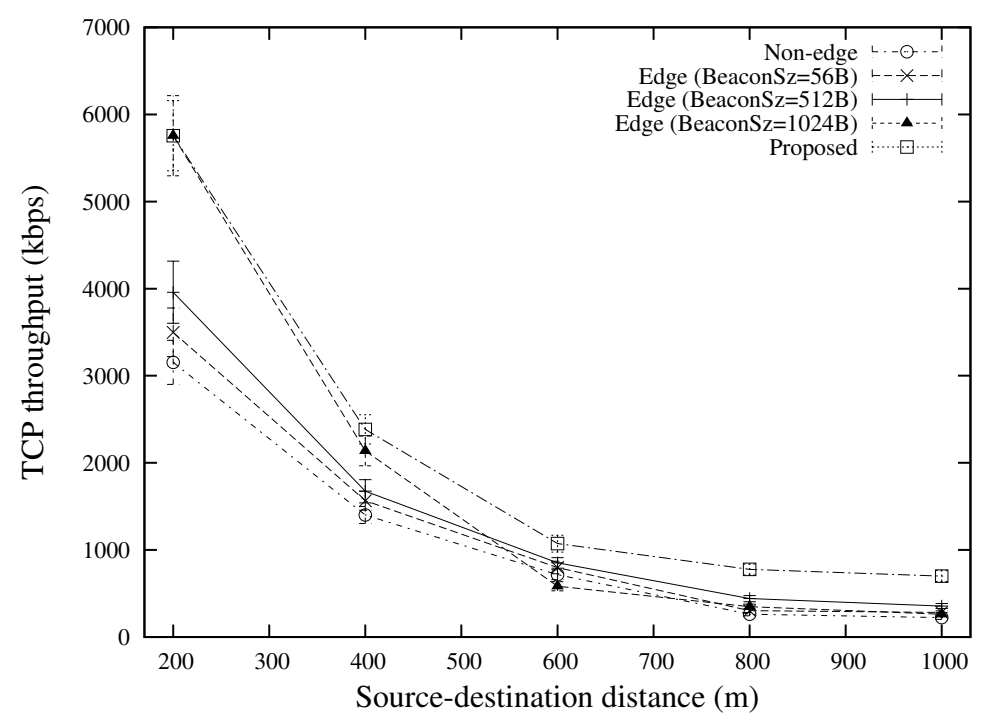

Figure 11. TCP throughput for various source-destination distances (the case of $20 \mathrm{TCP}$ flows).

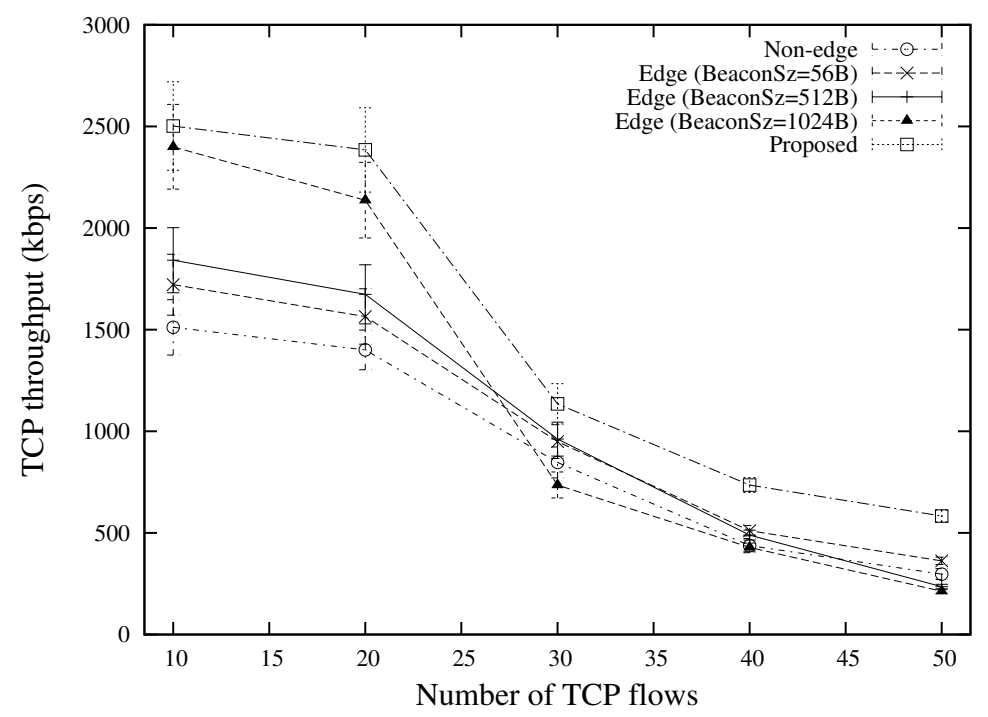

Figure 12. TCP throughput for various numbers of flows (the case of $400 \mathrm{~m}$ source-destination distance).

\subsection{Connection-Independent Unicast Flows}

Figure 13 shows the TCP throughput for various numbers of hops. By using the virtual multi-hop data delivery, the proposed scheme can achieve more than 20 percent improvement over other approaches in all situations ("Edge (BeaconSz $=56 \mathrm{~B})$ ", "Edge (BeaconSz $=512 \mathrm{~B})$ ", and "Edge (BeaconSz = $1024 \mathrm{~B})$ " do not use the virtual multi-hop data delivery). This is because the virtual multi-hop data delivery can result in a more efficient utilization of wireless resources by 
providing a higher congestion window size at each sender node as compared with the conventional end-to-end TCP approach.

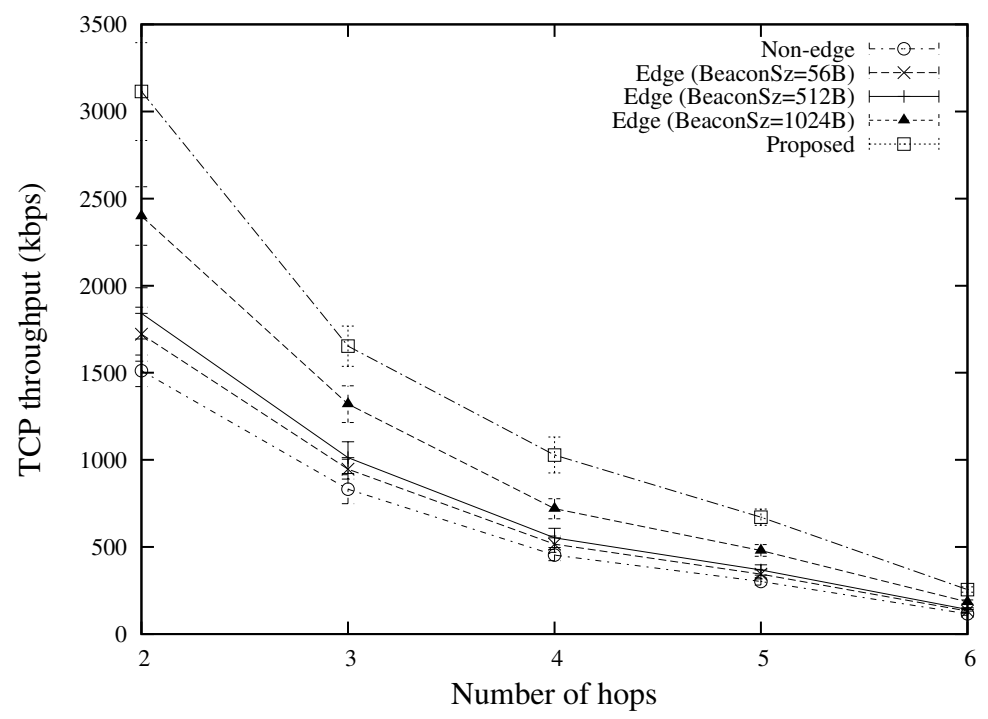

Figure 13. TCP throughput for various numbers of hops (the case of 10 TCP flows).

\section{Conclusions}

We proposed a context-aware edge-based routing scheme that supports both the broadcast applications and unicast applications. The scheme uses a context-aware approach to generate edge nodes which are used to forward the data packets for the purpose of reducing the number of contender nodes for the same wireless resources. Some content information, including the connection-dependency, communication type, and packet payload size, are considered in the route selection. A reinforcement learning-based approach is employed to optimize the last two-hop routes in order to improve the efficiency of the end-to-end communication. The simulation results show that the proposed scheme can achieve a significantly better performance as compared with other baseline approaches for different types of communications with different packet sizes.

Author Contributions: Conceptualization, C.A.; Methodology, C.A. and C.W.; Software, C.W.; Validation, T.Y., X.C. and Y.J.; Formal Analysis, T.Y., X.C. and Y.J.; Investigation, T.Y., X.C. and Y.J.; Writing-Original Draft Preparation, C.A. and C.W.; Writing-Review \& Editing, X.C.; Supervision, C.A.; Project Administration, C.A.; Funding Acquisition, C.W.

Funding: This research was supported in part by the open collaborative research program at National Institute of Informatics (NII) Japan (FY2018), the Telecommunications Advanced Foundation, JSPS KAKENHI Grant Number 16H02817, and 16K00121.

Conflicts of Interest: The authors declare no conflict of interest.

\section{References}

1. He, Y.; Zhao, N.; Yin, H. Integrated Networking, Caching, and Computing for Connected Vehicles: A Deep Reinforcement Learning Approach. IEEE Trans. Veh. Technol. 2018, 67, 44-55. [CrossRef]

2. Yuan, Q.; Zhou, H.; Li, J.; Liu, Z.; Yang, F.; Shen, S. Toward Efficient Content Delivery for Automated Driving Services: An Edge Computing Solution. IEEE Netw. 2018, 32, 80-86. [CrossRef]

3. Jutila, M. An Adaptive Edge Router Enabling Internet of Things. IEEE Internet Things J. 2016, 3, 1061-1069. [CrossRef]

4. Wang, K.; Yin, H.; Quan, W.; Min, G. Enabling Collaborative Edge Computing for Software Defined Vehicular Networks. IEEE Netw. 2018, PP, 1-6. [CrossRef]

5. Wisitpongphan, N.; Tonguz, O.K.; Parikh, J.S.; Mudalige, P.; Bai, F.; Sadekar, V. Broadcast storm mitigation techniques in vehicular ad hoc networks. IEEE Wirel. Commun. 2007, 14, 84-94. [CrossRef] 
6. Lai, W.; Ni, W.; Wang, H.; Liu, R.P. Analysis of Average Packet Loss Rate in Multi-Hop Broadcast for VANETs. IEEE Commun. Lett. 2018, 22, 157-160. [CrossRef]

7. Rodriguez, V.D.; Detournay, J.; Vinel, A.; Lyamin, N. An Approach for Receiver-Side Awareness Control in Vehicular Ad Hoc Networks. IEEE Trans. Intell. Transp. Syst. 2018, 19, 1227-1236. [CrossRef]

8. Alotaibi, M.M.; Mouftah, H.T. A Multi-Hop Broadcast Protocol for Emergency Message Dissemination in Urban Vehicular Ad Hoc Networks. IEEE Access 2017, 5, 4870-4886. [CrossRef]

9. Bi, Y.; Shan, H.; Shen, S.; Wang, N.; Zhao, H. A Multi-Hop Broadcast Protocol for Emergency Message Dissemination in Urban Vehicular Ad Hoc Networks. IEEE Trans. Intell. Transp. Syst. 2016, 17, 736-750. [CrossRef]

10. Kang, H.; Yoo, H.; Kim, D.; Chung, Y.-S. CANCORE: Context-Aware Network COded REpetition for VANETs. IEEE Access 2017, 5, 3504-3512. [CrossRef]

11. Nguyen, V.; Oo, T.Z.; Tran, N.H.; Hong, C.S. An Efficient and Fast Broadcast Frame Adjustment Algorithm in VANET. IEEE Commun. Lett. 2017, 21, 1589-1592. [CrossRef]

12. Xie, Y.; Ho, I. W.-H.; Magsino, E.R. The Modeling and Cross-Layer Optimization of 802.11p VANET Unicast. IEEE Access 2018, 6, 171-186. [CrossRef]

13. Zemouri, S.; Djahel, S.; Murphy, J. An Altruistic Prediction-Based Congestion Control for Strict Beaconing Requirements in Urban VANETs. IEEE Trans. Syst. Man Cybern. Syst. 2018, 1-16. [CrossRef]

14. Goudarzi, F.; Asgari, H.; Al-Raweshidy, H.S. Traffic-Aware VANET Routing for City Environments-A Protocol Based on Ant Colony Optimization. IEEE Syst. J. 2018. [CrossRef]

15. Yao, L.; Wang, J.; Wang, X.; Chen, A.; Wang, Y. V2X Routing in a VANET Based on the Hidden Markov Model. IEEE Trans. Intell. Transp. Syst. 2018, 19, 889-899. [CrossRef]

16. Darwish, T.S.J.; Bakar, K.A.; Haseeb, K. Reliable Intersection-Based Traffic Aware Routing Protocol for Urban Areas Vehicular Ad Hoc Networks. IEEE Intell. Transp. Syst. Mag. 2018, 10, 60-73. [CrossRef]

17. Zhang, X.; Cao, X.; Yan, L.; Sung, D.K. A Street-Centric Opportunistic Routing Protocol Based on Link Correlation for Urban VANETs. IEEE Trans. Mob. Comput. 2016, 15, 1586-1599. [CrossRef]

18. Sun, G.; Zhang, Y.; Liao, D.; Yu, H.; Du, X.; Guizani, M. Bus Trajectory-Based Street-Centric Routing for Message Delivery in Urban Vehicular Ad hoc Networks. IEEE Trans. Veh. Technol. 2018. [CrossRef]

19. Zhang, F.; Liu, H.; Leung, Y.-W.; Chu, X.; Jin, B. CBS: Community-Based Bus System as Routing Backbone for Vehicular Ad Hoc Networks. IEEE Trans. Mob. Comput. 2017, 16, 2132-2146. [CrossRef]

20. Seliem, H.; Shahidi, R.; Ahmed, M.H.; Shehata, M.S. Drone-Based Highway-VANET and DAS Service. IEEE Trans. Veh. Technol. 2018, 6, 20125-20137. [CrossRef]

21. Wu, C.; Ji, Y.; Ohzahata, S.; Kato, T. Can DTN Improve the Performance of Vehicle-to-roadside Communication? In Proceedings of the IEEE PIMRC, Hongkong, China, 30 August-2 September 2015; pp. 1934-1939.

22. Wu, C.; Yoshinaga, T.; Ji, Y. Context-aware unified routing for VANETs based on virtual clustering. In Proceedings of the IEEE PIMRC 2016 Workshops, Valencia, Spain, 4-8 September 2016; pp.1-6.

23. Wu, C.; Ohzahata, S.; Kato, T. VANET Broadcast Protocol Based on Fuzzy Logic and Lightweight Retransmission Mechanism. IEICE Trans. Commun. 2012, E95-B, 415-425. [CrossRef]

24. Zhang, L.; Wu, C.; Yoshinaga, T.; Chen, X.; Murase, T.; Ji, Y. Multihop Data Delivery Virtualization for Green Decentralized IoT. Wirel. Commun. Mob. Comput. 2017, 2017, 1-9. [CrossRef]

25. The Network Simulator-ns-2. Available online: http://www.isi.edu/nsnam/ns/ (accessed on 23 February 2018).

26. Khan, A.; Sadhu, S.; Yeleswarapu, M. A Comparative Analysis of DSRC and 802.11 over Vehicular Ad Hoc Networks; Project Report; University of California: Santa Barbara, CA, USA, 2009; pp. 1-8.

27. Krajzewicz, D.; Erdmann, J.; Behrisch, M.; Bieker, L. Recent Development and Applications of SUMO—Simulation of Urban MObility. Int. J. Adv. Syst. Meas. 2012, 5, 128-138.

28. TraNS (Traffic and Network Simulation Environment). Available online: http://trans.epfl.ch/ (accessed on 23 February 2018).

(C) 2018 by the authors. Licensee MDPI, Basel, Switzerland. This article is an open access article distributed under the terms and conditions of the Creative Commons Attribution (CC BY) license (http:/ / creativecommons.org/licenses/by/4.0/). 